\title{
Calidad de prescripción de montelukast en pacientes adultos: Estudio en tres farmacias comunitarias de la Comunidad Valenciana
}

\author{
$M^{a}$ Teresa Peris Molina', Fernando Ferrer Estrela ${ }^{2}, M^{a}$ Pilar D'Ocon Navaza ${ }^{3}$, Amalia Úbeda Pascual ${ }^{4}$ \\ 1. Farmacéutica comunitaria. Moncada (Valencia). 2. Farmacéutico comunitario. Mislata (Valencia). 3. Doctora en Farmacia. Catedrática de \\ Farmacología en la Universidad de Valencia. 4. Doctora en Farmacia. Profesora Titular de Farmacología en la Universidad de Valencia.
}

\section{PALABRAS CLAVE}

montelukast, asma,

rinitis alérgica,

glucocorticoides orales, recomendaciones de guias farmacológicas, régimen terapéutico

\section{ABREVIATURAS}

ATC: sistema de clasificación Anatómica Terapéutica Química. $\beta A C$ : betaagonista de acción corta.

CI: glucocorticoides inhalados.

GEMA: Guía Española para el Manejo del

Asma.

IC: intervalo de confianza.

n: número. semFYC: Sociedad Española de Medicina Familiar y Comunitaria. SNS: Sistema Nacional de Salud.

\section{KEYWORDS}

montelukast, asthma, allergic rhinitis, oral glucocorticoids, pharmacological guide recommendations, therapeutic regime

\section{RESUMEN}

Introducción: El objetivo de este trabajo es analizar la utilización de montelukast en adultos desde tres farmacias comunitarias.

Material y métodos: Estudio descriptivo, observacional en tres farmacias comunitarias (enero 2009 - febrero 2013) en pacientes mayores de 15 años que acuden a la farmacia con receta de montelukast. Los datos se recogen a través de encuesta (edad, patología, régimen terapéutico, duración del tratamiento, medicación asociada) y se valora la idoneidad de la prescripción según las indicaciones autorizadas y su adecuación a las guías de práctica clínica. Se remitió al médico, cuando se detectó un tratamiento inadecuado para la revisión del mismo.

Resultados: Se incluyen un total de 106 pacientes, de los cuales, el 26\% no padece asma y están tomando montelukast fuera de indicación. De los asmáticos, el $29 \%$ de los tratamientos pautados no se ajusta a las recomendaciones de las guías. Destaca tanto la elevada instauración de montelukast como tratamiento de inicio (59\% asmáticos, $63 \%$ no asmáticos), como el empleo de montelukast en monoterapia (16\% asmáticos, $41 \%$ no asmáticos).

Conclusiones: Se está haciendo un uso fuera de indicación de montelukast en pacientes no asmáticos con rinitis alérgica o bronquitis que podría derivar de la falta de claridad en las indicaciones de algunas guías. Además, en pacientes asmáticos, no siempre se está utilizando montelukast según las recomendaciones de las guías clínicas, lo que evidencia la necesidad de información clara a los profesionales sanitarios sobre el papel de montelukast en la terapéutica actual.

The Quality of Montelukast Prescriptions in Adult Patients.

A Study in three Community Pharmacies in the Valencia Region

\section{ABSTRACT}

Introduction: The objective of this piece of work is to analyse the use of Montelukast in adults from three community pharmacies.

Material and Methods: Descriptive, observational study in three community pharmacies (January 2009 - February 2013), in patients over the age of 15 who went to the pharmacy with a prescription for Montelukast. Their data was collected in a questionnaire (age, pathology, therapeutic regime, duration of the treatment, associated medication) and the suitability of the prescription was assessed according to the authorised indications and their adaptation to clinical practice guides. The patient was sent to the doctor when inadequate treatment was detected, to have it reviewed.

Results: 106 patients were included, of whom 26\% did not suffer from asthma and were taking Montelukast inappropriately. Of the asthmatics, $29 \%$ of the treatments prescribed did not adapt to the recommendations given in the guides. We would highlight the high level of establishment of Montelukast as an initial treatment (59\% asthmatics, $63 \%$ non-asthmatics), as well as the use of Montelukast in single-drug therapy (16\% asthmatics, $41 \%$ non-asthmatics).

Conclusions: Montelukast is being used incorrectly in non-asthmatic patients with allergic rhinitis or bronchitis which could lead to a lack of clarity in the indications in some guides. In addition, in asthmatic patients, Montelukast is not always being used according to the recommendations in the clinical guides, which shows the need for clearer information for health professionals about the role of Montelukast in current therapy.

Este trabajo fue presentado como comunicación en forma de póster en el V Congreso Nacional de Farmacéuticos Comunitarios de SEFAC en Barcelona, noviembre de 2012.

Recibido: 20-5-2013

Aceptado: $15-7-2013$

Disponible online: 1-3-2014
Financiación: Ninguna ajena.

Conflicto de intereses: Los autores declaran no existir ningún conflicto de interés en relación con el contenido del presente articulo. Cite este artículo como: Peris Molina MT, Ferrer Estrela F, D'Ocon Navaza MP, Úbeda Pascual A. Calidad de prescripción de montelukast en pacientes adultos: Estudio en tres farmacias comunitarias de la Comunidad Valenciana. Farmacéuticos Comunitarios. 2014 Mar 01;6(1):6-11. doi:10.5672/FC.2173-9218.(2014/Vol6).001.02 


\section{Introducción}

El asma es un importante problema de salud pública mundial. En España, la prevalencia se sitúa entre un 5 y un $10 \%$ de la población (1-4). La educación sanitaria de las personas asmáticas es la estrategia más útil y adecuada para alcanzar un buen control de la enfermedad.

En la actualidad, se realiza un tratamiento escalonado, ajustado según el nivel de gravedad del asma, que tiene como objetivo lograr y mantener el control de la enfermedad en la mayoría de los asmáticos (5).

Los antileucotrienos son fármacos utilizados en el tratamiento del asma, que llevan en el mercado más de diez años. A pesar de ser los glucocorticoides inhalados (CI) la piedra angular del tratamiento del asma persistente en niños y en adultos, la prescripción de antileucotrienos y, en concreto, montelukast, ha sobrepasado el lugar que les correspondería según la evidencia que actualmente se tiene de sus beneficios terapéuticos, lo que quizá se debe a su mayor facilidad de uso, comparados con otros antiasmáticos (6).

Varios ensayos han analizado el tratamiento con antileucotrienos frente a los CI en pacientes con asma persistente de leve a moderada. La mayoría de las comparaciones se han hecho entre montelukast y fluticasona (7). La conclusión general es que los CI son el tratamiento antiinflamatorio más eficaz y representan el fármaco de elección para el asma en cualquier grado. En general, la magnitud de los efectos antiinflamatorios de los antileucotrienos es menor que la obtenida con los corticoides (7), por lo que se considera el empleo de un antileucotrieno como segunda opción cuando no se puedan administrar los glucocorticoides inhalados (dificultad para utilizar la vía inhalada, problemas de candidiasis oral o intolerancia a los mismos) o cuando sea necesaria la vía oral (6).

En febrero de 2005, el laboratorio fabricante de montelukast incluyó como nueva indicación el alivio sintomático de la rinitis alérgica estacional en pacientes asmáticos. Aprobada esta indicación como tal, la práctica de uso puede llevar también, erróneamente, a utilizar montelukast en rinitis alérgica en cualquier tipo de pacientes, lo que, claramente, estaría fuera de indicación. De hecho, en la Guía Española para el Manejo del Asma (GEMA) (8), se establecen unas recomendaciones para el tratamiento de los pacientes con rinitis alérgica, ya que múltiples estudios epidemiológicos, fisiopatológicos y terapéuticos han demostrado la asociación entre rinitis y asma (9).

En esta guía se detalla la pauta terapéutica en población adulta, indicando que montelukast y zafirlukast son eficaces en el tratamiento de las rinitis y conjuntivitis alérgicas, y que se han mostrado igual de eficaces que los antihistamínicos, pero menos que los glucocorticoides tópicos nasales. Vemos, por tanto, que la guía no parece establecer una diferencia clara entre los pacientes con rinitis alérgica asmáticos, para los que sí estarian indicados los antileucotrienos, de los pacientes con rinitis no asmáticos, en los que la utilización de montelukast se produciría off-label, al no estar oficialmente aprobado su uso para el problema de salud en el cual se requiere o utiliza $(8,10)$.
El objetivo del presente trabajo es analizar, desde la farmacia comunitaria, la utilización de montelukast en pacientes asmáticos y no asmáticos y sus condiciones de uso asociado a otros medicamentos, valorando si el régimen terapéutico que lleva el paciente se ajusta al recomendado en las guías farmacoterapéuticas.

\section{Material y métodos}

Se trata de un estudio descriptivo, observacional, de utilización de medicamentos (estudio prescripción-indicación). La recogida de encuestas se realizó entre enero de 2009 y octubre de 2010 en tres farmacias de la Comunidad Valenciana (Moncada, Alginet y Mislata) a un total de 106 pacientes adultos.

Criterios de inclusión: pacientes mayores de 15 años que retiran una receta de montelukast en la farmacia comunitaria y acceden a responder a una encuesta. La medicación habitualmente es recogida por el propio paciente, al que se realizaba la encuesta tras el acto de dispensación.

Variables recogidas: datos sociodemográficos, patología para la que reciben montelukast, medicación adicional prescrita (que normalmente es retirada a la vez que montelukast con su correspondiente receta prescrita) y pautas de administración, otras patologías y grado de satisfacción de la medicación recibida.

Se ha valorado la idoneidad de la prescripción de montelukast según las indicaciones autorizadas en su ficha técnica (11). La adecuación de los medicamentos prescritos a los pacientes asmáticos se ha realizado consultando en la Guía Española del Asma 2009 (GEMA 2009) (8), la Guía de Práctica Clínica sobre el Asma (del Osakidetza/Servicio Vasco de Salud) (12) y la Guía de la British Society for Allergy and Clinical Immunology 2008 (13).

De igual forma se han valorado los tratamientos pautados en los pacientes no asmáticos (pacientes con rinitis o bronquitis) y que están recibiendo montelukast fuera de su indicación autorizada. Se han empleado las guías: Guía de Seguimiento Farmacoterapéutico sobre la Rinitis Alérgica 2005 (14), Guía de Bronquitis Aguda 2006 (de Guías Fisterra) (15), Guía de Rinitis 2003 (de Guías Fisterra) (16), Guía Rinitis semFYC 2007 (9), Rinitis Alérgica de Aula de la Farmacia 2007 (17) y la Guía de la British Society for Allergy and Clinical Immunology, 2008 (13).

\section{Resultados}

La media de edad de los 106 pacientes encuestados es de $48 \pm 19$ años (Intervalo de Confianza [IC] 95\%: 44-52), con un rango desde 16 años en adelante. Todos los pacientes recibían dosis diarias de montelukast de $10 \mathrm{mg}$, en forma de comprimidos recubiertos. Del total de pacientes incluidos en el estudio, 79 eran asmáticos (74\%) y 27 no (26\%), reflejando que aproximadamente la cuarta parte de pacientes a los que se realizó la encuesta no eran asmáticos y, sin embargo, estaban recibiendo montelukast en su farmacoterapia (figura 1). 


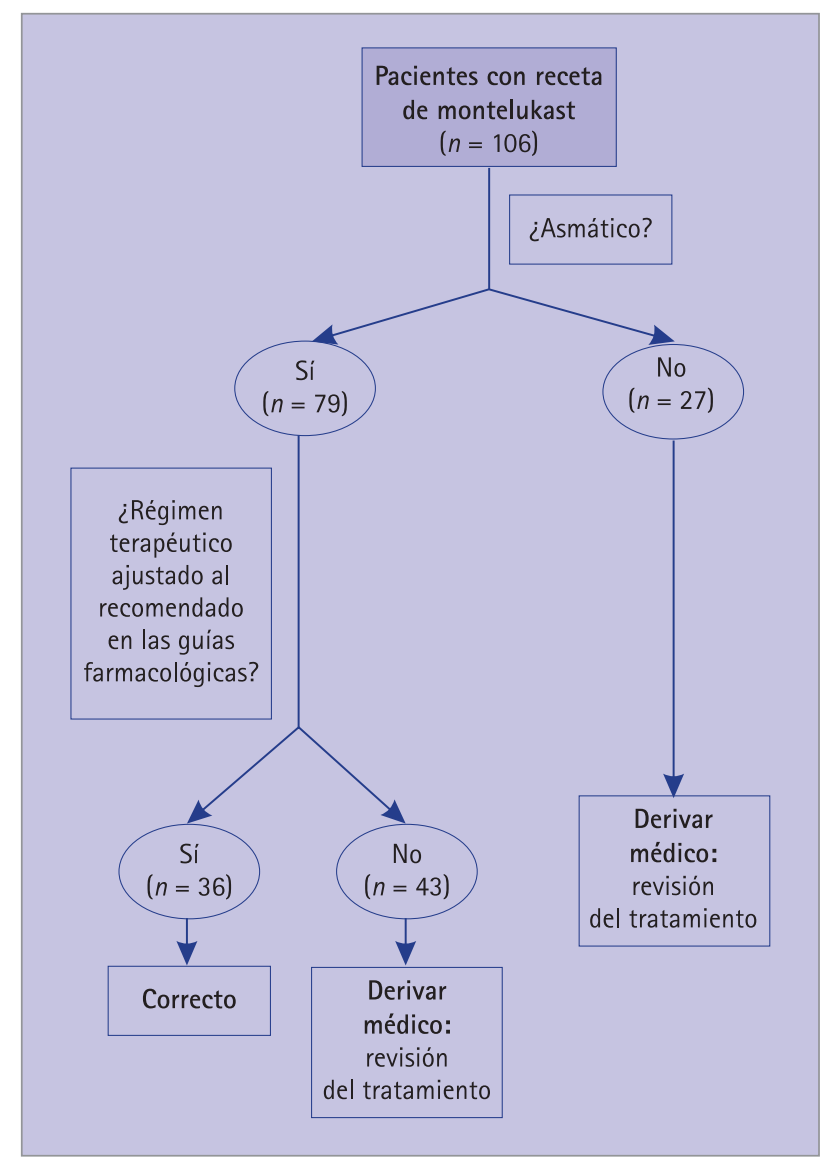

Figura 1 Algoritmo de actuación en los pacientes tratados con montelukast

La figura 2 muestra el tiempo de tratamiento con este fármaco, al $46 \%$ se les prescribe entre 2005 y 2008, y al $34 \%$, a partir de 2009, cuando se publica la Guía GEMA (8). Sólo el 20\% lo llevan pautado desde antes de 2005.

En la figura 3 se resumen las patologías de los pacientes para las que se ha prescrito montelukast, junto con la duración del tratamiento con este fármaco. La mayoría de los pacientes (49\%) son asmáticos que inician el tratamiento a partir de 2005, y a casi un tercio (32\%) se lo han prescrito en el año 2009 o con posterioridad. Un $26 \%$ de los pacientes no son asmáticos y, de ellos, un $22 \%$ padece rinitis alérgica y un $4 \%$ bronquitis. A la mayor parte $(78 \%)$ de los pacientes con rinitis

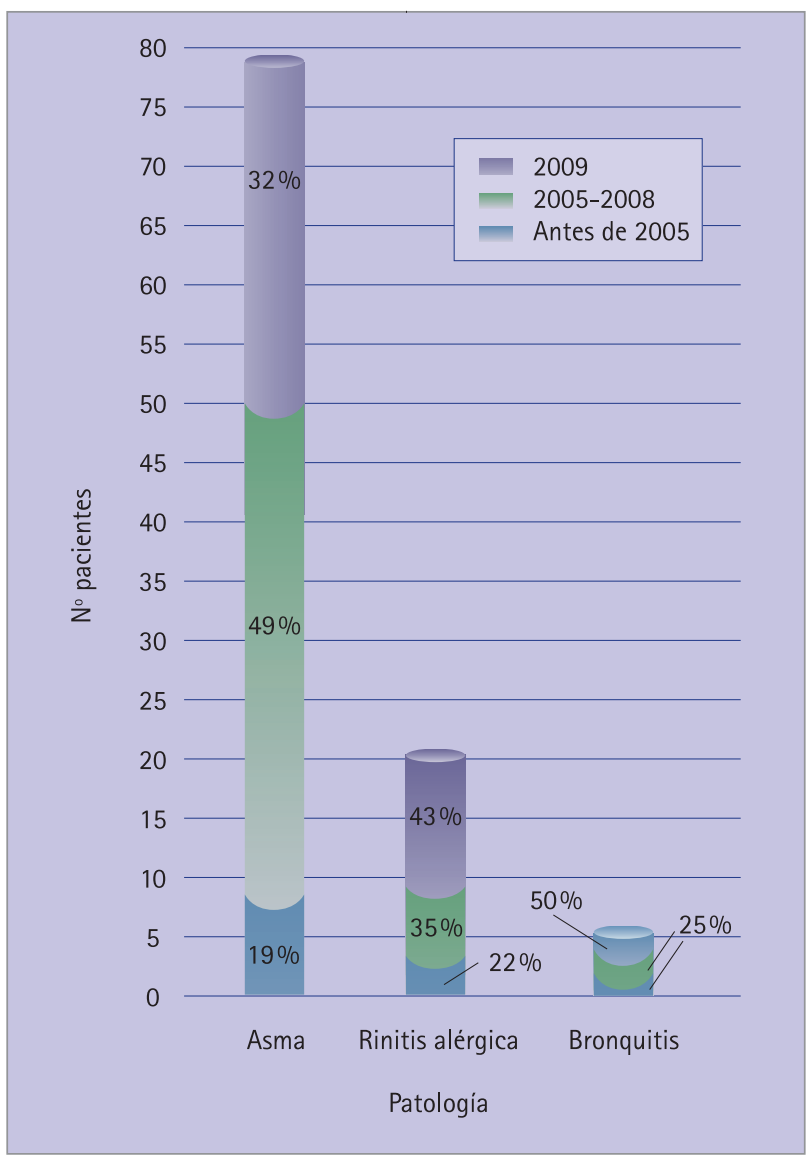

Figura 3 Clasificación de los pacientes según su patología y el tiempo de tratamiento con montelukast

alérgica se les ha pautado montelukast a partir de 2005, año en que se aprueba su indicación para la rinitis alérgica en pacientes asmáticos.

Evaluación de la calidad de prescripción de montelukast en pacientes adultos asmáticos

El grupo de 79 pacientes asmáticos presentó una edad media de $49 \pm 19$ años (IC 95\%: 45-53). El 84\% de los pacientes utiliza montelukast junto a otros fármacos como: agonistas $\beta 2$ adrenérgicos de acción corta (mayoritariamente salbutamol), glucocorticoides inhalados (budesonida de forma mayoritaria), antihistamínicos (desloratadina y cetirizina), glucocorticoides nasales (mayoritariamente
Figura 2 Tiempo de tratamiento con montelukast en los pacientes del estudio

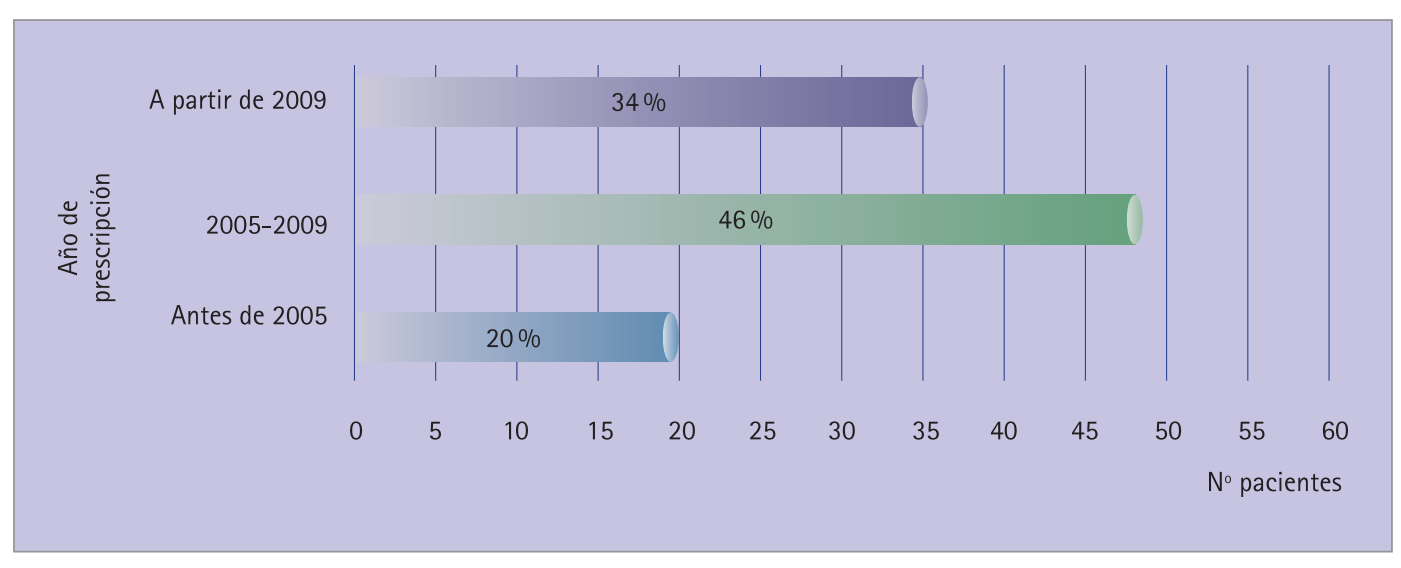




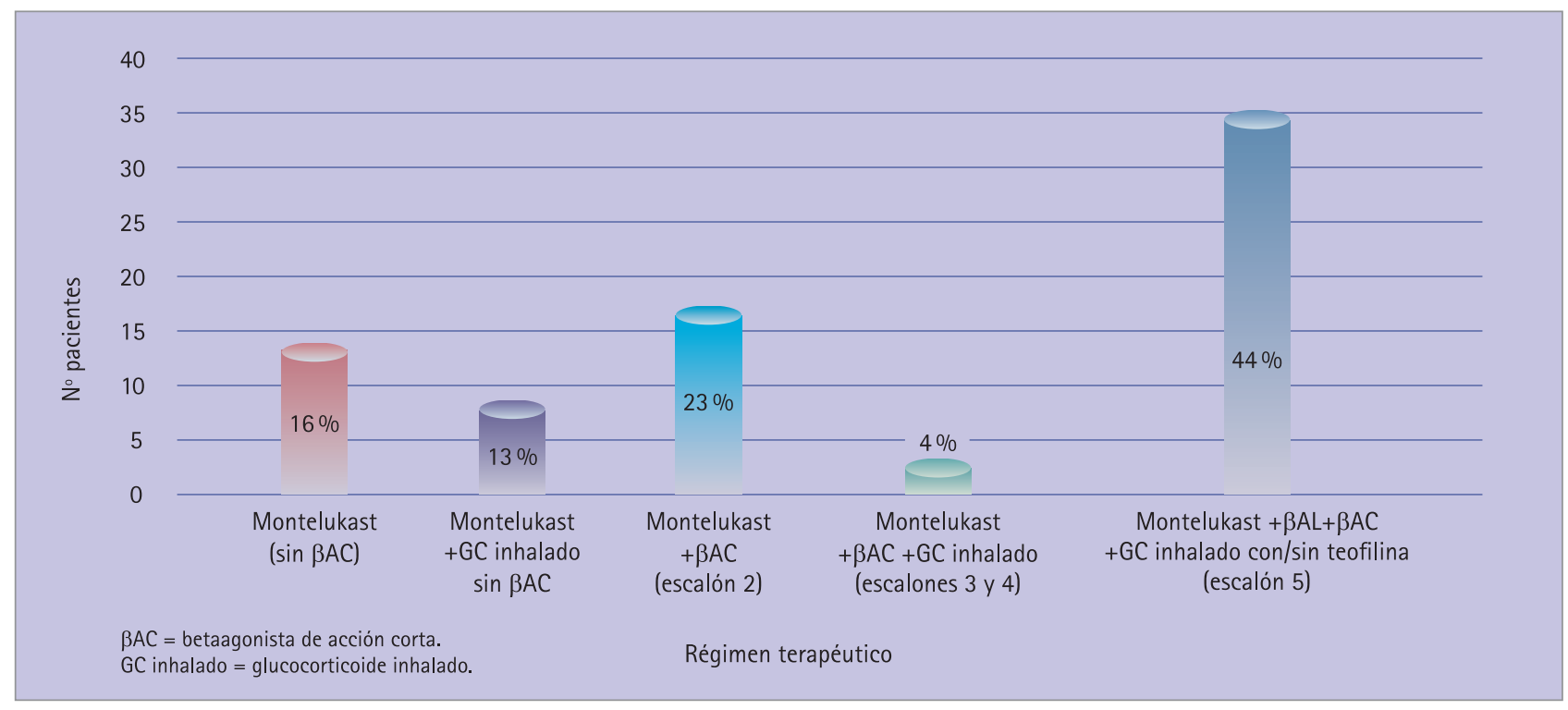

Figura 4 Régimen terapéutico seguido por los pacientes asmáticos

mometasona), y broncodilatadores (bromuro de ipratropio como más empleado) y vacunas.

El régimen terapéutico se resume en la figura 4 , en la que se agrupan los pacientes según los escalones terapéuticos recomendados en la Guía GEMA 2009 (8). Destaca que 13 pacientes (16\%) utilizan montelukast en monoterapia, situación que no corresponde con ninguno de los escalones recomendados. También es destacable que 10 (13\%) pacientes utilizan montelukast junto con CI, sin incluir en su tratamiento un agonista $\beta 2$ adrenérgico de acción corta como medicación de rescate. Un 71 \% de los pacientes sigue las recomendaciones terapéuticas de las guías.

El 59\% de los pacientes asmáticos ha iniciado el tratamiento del asma con montelukast, a pesar de que no es la primera opción recomendada. De los que llevaban tratamiento previo, al $14 \%$ se le añade el montelukast al tratamiento anterior, mientras que en el $27 \%$ se prescribe montelukast para sustituir a otra medicación. Los fármacos más sustituidos fueron antihistamínicos, agonistas betaadrenérgicos y vacunas.

\section{Prescripción de montelukast en adultos no asmáticos}

La edad media de este grupo de 27 pacientes es de $45 \pm 18$ años (IC 95\%: 41-49). La mayor parte de estos pacientes está tomando montelukast para la rinitis alérgica (85\%) sin ser asmáticos, y el resto, para la prevención de bronquitis. En cuanto a su régimen terapéutico, 11 pacientes ( $41 \%$ ) emplean montelukast en monoterapia. En el empleo asociado encontramos mayoritariamente agonistas betaadrenérgicos (empleado en las crisis como fármaco de rescate), antihistamínicos, glucocorticoides, corticoides nasales, mucolíticos, xantinas, broncodilatadores y vacunas.

Un $37 \%$ de los pacientes no asmáticos llevaba un tratamiento previo con otros principios (antihistamínicos, betaadrenérgicos, glucocorticoides, broncodilatadores beta y vacunas), retirándoles la medicación previa al $90 \%$ de ellos y adicionándoles montelukast a los restantes.

\section{Discusión}

Montelukast fue autorizado en 2001, pero es en febrero de 2005 cuando se añade la nueva indicación de "tratamiento en rinitis alérgica en pacientes asmáticos" y, en la Guía GEMA 2009 (8), aparece como recomendación en alérgicos. Ello contrasta con otras guías clínicas que no incluyen a montelukast como primera opción en este caso, sino que el tratamiento de elección para estos pacientes son los corticoides nasales y los antihistamínicos, más eficaces y de menor coste $(9,10,13-18)$. Si analizamos en nuestra muestra de población la fecha de inicio del tratamiento con montelukast, observamos que el $80 \%$ de las prescripciones se han hecho por un periodo a partir de 2005, lo que indica que el número de pacientes se incrementa significativamente a partir de esta fecha, en la que aparece la nueva indicación de uso de montelukast en la ficha técnica "asmáticos con rinitis alérgica".

Dentro de este $80 \%$, un $42 \%$ de los pacientes ha iniciado el tratamiento a partir de 2009, coincidiendo con la publicación de la Guía GEMA (8), en la que se recomienda montelukast en pacientes con rinitis, sin marcar claramente una diferencia en el tratamiento de los pacientes con rinitis asmáticos de los no asmáticos. Este aumento que encontramos en nuestra población también viene reflejado en los datos de consumo del Sistema Nacional de Salud (SNS) extraído de los boletines de Información Terapéutica del SNS, donde se detallan los subgrupos ATC (sistema de clasificación anatómica, terapéutica, química) de mayor consumo a nivel nacional, siendo el gasto nacional correspondiente al grupo de antileucotrienos de 79,9 millones de $€$ en 2006 (1.833,3 miles de envases) (19), 92,14 millones de $€$ en 2007 (2.125,72 miles de envases) (20), 101,20 millones de $€$ en 2008 (2.335,5 miles de envases) (21), 109,8 millones de $€$ en 2009 (2.537,6 miles de envases) (22), y 117,8 millones de $€$ en 2010 (2.722,8 miles de envases) (23), lo que refleja un aumento creciente en su prescripción.

El incremento que se produce en 2009 se observa tanto en asmáticos como en pacientes no asmáticos con rinitis alérgica o bronquitis, en los que montelukast no 
está indicado. El uso de montelukast para indicaciones no aprobadas en ficha técnica no se considera adecuado ni siquiera como uso compasivo, ya que no hay evidencias suficientes de la eficacia del montelukast en estos procesos (24-26).

Cuando analizamos la adecuación del régimen terapéutico de los pacientes asmáticos a las recomendaciones de las guías consultadas encontramos que en la mayoría de los casos sí se corresponde con las indicaciones, pero detectamos una inadecuación del tratamiento en un $22 \%$ de los pacientes que reciben montelukast en monoterapia o bien combinado con $\mathrm{CI}$, pero no están utilizando un agonista ( $\beta 2$-adrenérgico de acción corta como medicación de rescate.

El uso de montelukast en monoterapia no está recomendado por ninguna guía, y esta situación puede deberse a varios motivos, entre los que podemos considerar que el paciente no conozca bien su tratamiento, que no tenga una buena adherencia al mismo o que no esté siendo adecuadamente tratado.

Considerando estos tres supuestos, el farmacéutico comunitario puede mejorar el tratamiento de los pacientes asmáticos, por un lado, informando al paciente sobre su tratamiento, incidiendo tanto en el cumplimiento terapéutico como en el correcto uso de cada uno de los fármacos prescritos y, por otro lado, controlando que el régimen terapéutico se ajuste al recomendado por las guías clínicas vigentes según la patología referida.

En cuanto a la población que está recibiendo montelukast sin padecer asma, sería importante la labor del farmacéutico comunitario identificando a estos pacientes $\mathrm{y}$, en colaboración con su médico, valorar la posibilidad de cambiar a tratamientos de primera elección según las guías farmacoterapéuticas consultadas.

La información obtenida a través de las encuestas puede no reflejar con exactitud el diagnóstico de las enfermedades y la medicación prescrita, aunque sí refleja los conocimientos que tiene el paciente de su medicación, y que en muchos casos no sigue las pautas recomendadas por las guías e indica un tratamiento inadecuado del asma.

\section{Conclusiones}

Los resultados indican que se está haciendo un uso fuera de indicación del montelukast en pacientes no asmáticos con rinitis alérgica o bronquitis, que podría derivar de la falta de claridad en las indicaciones de la Guía Española para el Manejo del Asma (2009). Además, en pacientes asmáticos, no siempre se está utilizando el montelukast según las recomendaciones de las guías clínicas. Dado que el tratamiento con montelukast es notablemente más costoso que los tratamientos convencionales, además de disponer de menos experiencia clínica en su uso comparado con las alternativas tradicionales, se considera la postura más racional la que adoptan la mayoría de las guías clínicas consultadas, siendo deseable una recomendación clara en la Guía Española del Asma respecto a su uso en rinitis no asmática. Por todo ello, se evidencia la necesidad de información clara a los profesionales sanitarios sobre el papel de montelukast en la terapéutica actual.

\section{Referencias bibliográficas}

1. Grupo Español del Estudio Europeo del Asma. Estudio Europeo del Asma: Prevalencia de síntomas relacionados con el asma en cinco áreas españolas. Med Clin (Barc). 1995;104:487-92.

2. Grupo Español del Estudio Europeo del Asma. Estudio Europeo del Asma: Prevalencia de hiperreactividad bronquial y asma en adultos jóvenes de cinco áreas españolas. Med Clin (Barc). 1996;106:761-7.

3. European Community Respiratory Health Survey (ECRHS). Variations in the prevalence or respiratory symptoms, self-reported asthma attacks, and use of asthma medication in the ECRHS. Eur Respir J. 1996;9:687-95. doi:10.1183/090 31936.96.09040687

4. International Study of Asthma and Allergies in Childhood (ISAAC) Steering Committee. Worldwide variation in prevalence of symptoms of asthma, allergic rhinoconjunctivitis, and atopic eczema: ISAAC. Lancet. 1998;351:1225-32. doi:10.1016/S0140-6736(97)07302-9

5. De Andrés Jácome J, Iñesta García A. Estudio prospectivo sobre el impacto de un servicio de Atención Farmacéutica Comunitaria en personas asmáticas. Rev Esp Salud Pública. 2003;77:393-403.

6. Sistema de Salud de Navarra [Internet]. Lugar de los antileucotrienos en la terapéutica infantil. Boletín de Información terapéutica de Navarra. Volumen 17, $N^{\circ}$ 1. 2009 [Acceso 25 de abril de 2012]. Disponible en: http://www.navarra.es/ home_es/Temas/Portal+de+la+Salud/Profesionales/Documentacion+y+publicaciones/Publicaciones+tematicas/Medicamento/BIT/Vol+17/BIT+17+1.htm

7. Knorr B, Franchi LM, Bisgaard H, Vermeulen JH, LeSoeuf P, Santanello N, et al. Montelukast, leukotriene receptor antagonist, for the treatment of persistent asthma in children aged 2 to 5 years. Pediatrics. 2001;108(3):E48. doi:10.1542/peds.108.3.e48

8. GEMA. Guía de Práctica Clínica sobre el Manejo del Asma [Internet]. 2009 [Acceso 20 de marzo de 2013]. Disponible en: http://www.gemasma.com/images/stories/GEMASMA/Documentos/GEMA\%202009/index.html

9. Vilaseca Canals J, Espinas Boquet J, editores. Guía Terapéutica en Atención Primaria basada en la evidencia. 3a edición. Barcelona: SEMFYC Ediciones; 2007.

10. Sistema de Salud del País Vasco [Internet]. Informe de evaluación: Montelukast. Comité de evaluación de nuevos medicamentos. Gobierno Vasco $\mathrm{N}^{\circ}$ 109. 2006 [Acceso 25 de enero de 2013]. Disponible en: http://www.osakidetza.euskadi.net/contenidos/informacion/cevime_nuevo_medicamento/es_nme/adjuntos/montelukast_informe.pdf

11. Agencia Española del Medicamento [Internet]. Fichas técnicas de medicamentos [Acceso 25 de abril de 2013]. Disponible en: http://www.aemps.gob.es/cima/especialidad.do? metodo=verFichaWordPdfacodigo $=742858$ formato $=$ pdfaformulario=FICHAS

12. Servicio Vasco de Salud. Guía de Práctica Clínica sobre Asma [Internet]. 2005 [Acceso 20 de marzo de 2013]. Disponible en: http://www.osakidetza.euskadi.net/r85-pkpubl02/ es/contenidos/informacion/osk_publicaciones/es_publi/adjuntos/guias/asma.pdf

13. BSACI. Guidelines for the management of allergic and non-allergic [Internet]. 2008 [Acceso 20 de marzo de 2013]. Disponible en: http://www.bsaci.org/Guidelines/bsaci-guidelines

14. Grupo de investigación en Atención Farmacéutica. Guía de Seguimiento Farmacoterapéutico sobre la Rinitis Alérgica. Granada: Universidad de Granada; 2005.

15. Fisterra. Guía Clínica de Bronquitis aguda de Fisterra [Internet]. 2006 [Acceso 20 de marzo de 2013]. Disponible en: http://www.agamfec.com/pdf/CADERNOS/VOL13/VOL13_3/ 06_Guias_de_Fisterra_B.pdf 
16. Fisterra. Guía Clínica de la Rinitis en Atención Primaria de Fisterra [Internet]. 2003 [Acceso 20 de marzo de 2013]. Disponible en: http://www.ugr.es/ cts131/esp/guias/GUIA_RINITIS.pdf

17. Aula de la Farmacia. Rinitis Alérgica: Actualizaciones [Internet]. 2007 [Acceso 20 de marzo de 2013]. Disponible en: http://www.auladelafarmacia.com/af/documentos/rinitis. pdf

18. Sistema de Salud del País Vasco [Internet]. ¿Montelukast en el tratamiento de la bronquiolitis? Infac: Información Terapéutica de la Comarca. Volumen 12, N 6. 2004 [Acceso 24 de abril 2013]. Disponible en: http://www.osakidetza.euskadi.net/r85-pkcevi03/es/contenidos/informacion/ cevime/es_cevime/r01hRedirectCont/contenidos/informacion/cevime_infac/es_cevime/adjuntos/infac_v12_n6.pdf

19. Información Terapéutica del Sistema Nacional de Salud [Internet]. Subgrupos ATC de mayor consumo en el Sistema Nacional de Salud en 2006. Inf Ter Sist Nac Salud. 2007;31(4):130-5. [Acceso 20 de abril de 2013]. Disponible en: http://www.msc.es/biblioPublic/publicaciones/docs/ vol31_4SubgruposATC2006.pdf

20. Información Terapéutica del Sistema Nacional de Salud [Internet]. Subgrupos ATC de mayor consumo en el Sistema Nacional de Salud en 2007. Inf ter Sist Nac Salud. 2008;32(4):128-132. [Acceso 20 de abril de 2013]. Disponible en: http://www.msc.es/biblioPublic/publicaciones/recursos_propios/infMedic/docs/vol32_4NoticiasTemasInteres.pdf

21. Información Terapéutica del Sistema Nacional de Salud [Internet]. Subgrupos ATC de mayor consumo en el Sis- tema Nacional de Salud en 2008. Inf ter Sist Nac Salud. 2009;33(4):120-2. [Acceso 20 de abril de 2013]. Disponible en: http://www.msc.es/biblioPublic/publicaciones/ recursos_propios/infMedic/docs/vol33_4NotasInteresSubgruposATC.pdf

22. Información Terapéutica del Sistema Nacional de Salud [Internet]. Subgrupos ATC de mayor consumo en el Sistema Nacional de Salud en 2009. Inf ter Sist Nac Salud. 2010;34(3):89-92. [Acceso 20 de abril de 2013]. Disponible en: http://www.msc.es/biblioPublic/publicaciones/recursos_ propios/infMedic/docs/vol34n3SubgrATCppiosActivos.pdf

23. Información Terapéutica del Sistema Nacional de Salud [Internet]. Subgrupos ATC de mayor consumo en el Sistema Nacional de Salud en 2010. Inf ter Sist Nac Salud. 2011;35(4):124-8. [Acceso 20 de abril de 2013]. Disponible en: http://www.msc.es/biblioPublic/publicaciones/recursos_ propios/infMedic/docs/SubgruposATCvol35n4.pdf

24. Bousquet J, Khaltaev N, Cruz AA, Denburg J, Fokkens WJ, Togias A, et al. Allergic Rhinitis and its Impact on Asthma (ARIA) 2008 Update. Allergy. 2008;63(Supl.86):8160.

25. Togias A. Rhinitis and asthma: evidence for respiratory system integration. J Allergy Clin Immunol. 2003;111(6):117183; quiz 1184. doi:10.1067/mai.2003.1592

26. Bisgaard H, Flores-Nunez A, Goh A, Azimi P, Halkas A, Malice MP, et al. Study of Montelukast for the treatment of respiratory symptoms of post-respiratory syncytial virus bronchiolitis in children. Am J Respir Crit Care Med. 2008;178:854-60. doi:10.1164/rccm.200706-9100C 